\title{
D esigualdades de género durante la transición a la vida adulta. Estudio exploratorio ${ }^{1}$
}

\author{
D aniel La Parra C asado
}

Universitat d'Alacant. D epartament de Salut Pública

Ap. Correus 99. E-03080 Alacant (Spain)

D aniel.LaParra@ua.es

\section{Resumen}

El estudio de las diferencias de género a lo largo del ciclo de vida proporciona numerosas claves para la comprensión de las desigualdades sociales entre hombres y mujeres. Los años de paso del sistema educativo al mundo del trabajo y de la familia de origen a la independencia familiar influyen en la posterior situación profesional de las mujeres y en el tipo de responsabilidades familiares (actividades reproductoras) que irán asumiendo las nuevas generaciones de mujeres españolas. La investigación analiza la importancia de los padres y madres en la definición de las trayectorias de género e introduce toda una serie de sugerencias sobre como las determinaciones familiares son interdependientes con otros procesos sociales, en concreto, la evolución del sistema educativo y del mercado de trabajo.

Palabras clave: ciclo de vida, género, familia, jóvenes, mercado de trabajo, sistema educativo, socialización.

Abstract. $G$ ender differences in the transition from youth to adulthood

The study of gender differences throughout the life course is essential to understand the social differences between women and men. The transitions from the educational system to the labor market and from family dependence to family independence determine women occupational careers and women rôles in the family. This research focuses on the parental socialization and the social construction of life trajectories. The conclusion which emerges is that the parental socialization is interdependent with the evolution of the labor market and the educational system.

Key words: educational system, familiy, gender, labor market, life-cycle, socialization, youth.

1. Este trabajo fue posible gracias a la participación y colaboración de las personas entrevistadas. Ellos pueden ser considerados los verdaderos autores de la investigación. Les agradezco especialmente su valiosa contribución. Agradezco igualmente la ayuda y dirección de los profesores José M aría Tortosa Blasco y T hierry Blöss en la realización de esta investigación que condujo a la lectura de la tesis de licenciatura: Género y transición a la vida adulta. La construcción social de la identidad familiar y profesional en septiembre de 1998. 


\section{Sumario}

$\begin{aligned} \text { Introducción } & \text { Resultados obtenidos } \\ \text { Consideraciones teóricas } & \text { Bibliografía } \\ \text { Metodología } & \end{aligned}$

\section{Introducción}

El objeto de estudio de la presente investigación son las desigualdades de género durante la transición a la vida adulta. Se trata, por tanto, de una investigación sobre las diferencias entre hombres y mujeres en el paso del sistema educativo al mundo del trabajo y de la familia en la que nacen a la independencia familiar. M ás concretamente se centra en el papel de la socialización familiar en la definición de trayectorias diferentes entre hijos e hijas durante la entrada en la vida adulta. Es decir, en la influencia de los padres y madres en la construcción social de la identidad familiar y profesional de género. Este objeto se aborda a partir de una población de estudio muy específica. Se ha entrevistado a 20 jóvenes de entre 30 y 32 años, que estudiaron bachillerato, y a sus padres (8) residentes, unos y otros, en un barrio de clase trabajadora (EI Pla, Alicante). Las entrevistas son de carácter retrospectivo y recogen los relatos sobre sus trayectorias escolares, familiares y profesionales desde la edad en la que realizaban sus estudios secundarios hasta la actualidad.

Por su construcción metodológica, la investigación tiene un carácter exploratorio, lo que no impide que se haya dado una gran importancia a la parte teórica. Se ha acudido al trabajo de campo como punto de partida para la obtención de nuevas ideas, conceptos e hipótesis, y no para contrastar hipótesis con los datos empíricos. Ello supone que las conclusiones son presentadas como propuestas para la comprensión del objeto sociológico tratado y no como respuestas o hipótesis validadas.

El objetivo perseguido es mostrar que las trayectorias de vida de género se construyen socialmente y precisar qué papel cumple la familia, los padres y las madres, en dicha construcción.

Como en toda investigación, una vez precisado el tema objeto de estudio, era necesario proceder a su desarrollo con el objetivo de encontrar explicaciones tan alejadas como fuese posible de la percepción subjetiva. En este sentido, como es conocido, se disponía de tres instrumentos principales de objetivación: la teoría, la metodología y el trabajo de campo. En todo momento se evitó que uno de ellos tomara la primacía sobre los demás.

U na de las cuestiones directrices que ha impulsado la elaboración del trabajo puede plantearse como sigue: ipor qué la posición, en principio ventajosa, de las mujeres en el sistema educativo se transforma en una situación de desventaja en el mundo laboral? 
Si consideramos el tramo de edad entre los 16 y los $29^{2}$ años, el más afectado, por lo demás, por el desempleo, observaremos que mientras que las mujeres tienen unos niveles de formación más elevados que los hombres de su edad, éstos últimos son los que se hallan en una condición más favorable en el mercado de trabajo (menor desempleo, mayor tasa de actividad, menor incidencia de la contratación precaria, mejores salarios, menor incidencia de la contratación a tiempo parcial). Estos hechos nos muestran que la relación entre formación y empleo no es tan directa como se podría pensar en un principio. Sin embargo, cada vez que se plantea el problema del desempleo se buscan respuestas relacionadas con la formación. D escubrir que la relación entre formación y empleo está mediatizada por factores como el género plantea todo tipo de interrogantes acerca de la función que cumple el sistema educativo en nuestras sociedades. Es habitual justificar las desigualdades sociales con explicaciones basadas en principios meritocráticos. En concreto, se suele señalar que la mejor o peor formación se corresponde con una mejor o peor situación social. Sin embargo cuando descubrimos que las mujeres, a pesar de estudiar durante un periodo de tiempo superior y ser más numerosas entre los que estudian bachillerato y estudios universitarios, presentan peores situaciones en el mercado de trabajo, las explicaciones meritocráticas se tambalean. Se impone la reflexión sobre cuáles son los mecanismos que permiten que se produzca la inversión de la posición social de la mujer según se encuentre en el sistema educativo o en el mundo laboral. D e dicha reflexión surgirán otro tipo de cuestiones: ¿qué otros grupos sociales se ven afectados por fenómenos similares?, ¿qué valor se puede conceder al sistema educativo como mecanismo de ascensión social? En definitiva, el origen de las desigualdades sociales debe buscarse también fuera del sistema educativo. 0 mejor: el origen de las desigualdades sociales puede depender de la forma en que las diferentes instituciones actúan conjuntamente en su construcción. Aquí se tratará de describir algunas de esas relaciones entre educación, familia y mercado de trabajo.

\section{C onsideraciones teóricas}

Por lo que respecta al procedimiento teórico se afrontaban varios problemas. En primer lugar, el punto de vista adoptado presenta al gunas novedades. U na de esas novedades consistía en proponer la entrada en la vida adulta, esto es, el paso del sistema educativo al mundo del trabajo, como un momento clave en la construcción de la identidad de género. N ormalmente las investigaciones otorgan un mayor valor a la socialización primaria. En esta línea lo más habitual es destacar como la infancia o la adolescencia (Erikson, 1968) influyen en la configuración de la identidad de la persona. Sin embargo, la obra 
de Berger y Luckmann (1968) se enmarca ya dentro de una tendencia a enfatizar la importancia de la socialización secundaria en la construcción social de la realidad y en la creación de las condiciones para la acción social de los actores. A mi modo de ver, la elección de este período del ciclo de vida se justifica porque es cuando se inicia la vida laboral cuando cualquier persona empieza a construir su identidad profesional. También porque es cuando se comienza a formar una familia cuando se empiezan a adoptar los diferentes roles familiares. Además es precisamente en estos dos aspectos donde el cambio de la situación de las mujeres está siendo mucho más importante, por un lado, el crecimiento del trabajo asalariado femenino y, por otro, la transformación de las estructuras de parentesco (de las formas de familia).

U na segunda novedad teórica viene dada por la consideración de nuevos fenómenos sociales que pueden estar influyendo y transformando la forma tradicional de entender las relaciones entre generaciones. En este sentido, tradicionalmente se ha atribuido una gran importancia a la familia en la interiorización de las diferencias entre hombres y mujeres. Así, por ejemplo, desde la psicología (empezando por Freud), se ha destacado el papel que cumplen la figura paterna y materna en la formación de la personalidad del individuo. También tradicionalmente desde la sociología (D urkheim, 1922) se ha insistido sobre la importancia de la transmisión de valores y de representaciones sociales de padres a hijos. La perspectiva adoptada no pretende negar esa influencia. Sin embargo, sí pretende enriquecerla (y en algunos casos relativizarla) con la consideración de nuevos fenómenos sociales que pueden, como decía, estar influyendo y transformando la forma tradicional de entender las relaciones entre generaciones. Por un lado, debido al lugar cada vez más importante que está ocupando el sistema educativo en las relaciones familiares. La escuela se introduce de forma cada vez más clara en el interior de la familia. Por otro, porque el mercado de trabajo se está constituyendo en una de las referencias claves para la explicación de los procesos de formación de identidad (Blöss, 1997).

D eacuerdo con D ubar (1991; 119-122), a partir de la crisis económica y durante la actual etapa de reestructuración económica el periodo de entrada en la edad adulta ha ido adquiriendo una mayor importancia en la formación de la identidad de los jóvenes. La disminución de la oferta de empleo, la precariedad, el desempleo han provocado que los individuos sólo puedan definirse profesionalmente una vez han atravesado toda una serie de circunstancias en el mercado de trabajo. M ientras que con anterioridad, el haber estudiado una determinada carrera permitía vislumbrar una actividad profesional dentro del campo de conocimientos que se habían adquirido en la misma, en la actualidad serán los pasos que se sigan dentro del mercado de trabajo los que definan el tipo de actividad laboral que se desarrollará finalmente.

Todas estas novedades, y algunas otras, en el planteamiento teórico han provocado que no existiera un modelo teórico completo con normas y reglas bien establecidas a propósito del objeto de estudio. Sin dicho marco teórico no se podían elaborar hipótesis y acudir al trabajo de campo para verificar- 
las. D e hecho se debía acudir al trabajo de campo con una intención bien distinta, una intención de tipo exploratorio, es decir, tratar a partir de las entrevistas de descubrir todos aquellos el ementos que puedan ayudar a comprender las transformaciones sociales que se vienen comentando. Esto es, de lo que se trataba era que todo lo que se dijera en la investigación partiera de la información recabada en las entrevistas.

\section{M etodología}

El diseño metodológico se encaminó a conseguir una técnica de recogida de información capaz de manejar el ciclo de vida y las trayectorias escolares, familiares y profesionales. En este sentido, se consideró que las entrevistas en profundidad de carácter retrospectivo eran la técnica que más convenía. Sobre todo si se tiene en cuenta que estas entrevistas se mantuvieron tanto con los jóvenes que eran objeto de estudio como con sus padres.

La comprensión de los resultados está íntimamente ligada a las características concretas de las personas entrevistadas.

a) Se entrevistó a personas que tenían entre 30 y 32 años en el momento de la entrevista, con ello se pretendía que tuvieran una edad suficiente para que la llamada «transición a la vida adulta» hubiera sido completada 0 estuviera a punto de hacerlo. Esto es, que hubieran entrado en el mercado laboral y hubieran iniciado una nueva vida familiar independiente de la familia de origen.

b) Los entrevistados nacieron entre 1966 y 1968. Este dato resulta de gran interés para comprender los particulares momentos históricos en los que ambas generaciones construyeron sus trayectorias de vida.

c) Los entrevistados fueron seleccionados por el hecho de haber estado matriculados en un instituto de bachillerato, esta circunstancia hace suponer que han seguido una larga escolarización. Además, al escoger un grupo de jóvenes que a la edad de 16 años comparte una situación bastante similar (mismo tipo de estudios, origen social, entorno), permite percibir como se van diversificando sus trayectorias a partir de un punto de origen común.

d) Por último, todos los jóvenes entrevistados durante los años del instituto vivían en un barrio que está fundamental mente ocupado por empleados en el comercio, obreros de la construcción y la pequeña industria y trabajadores en servicios, es decir, por una clase trabajadora poco cualificada que percibe salarios modestos, pero normal mente estables.

\section{Resultados obtenidos}

Antes de iniciar el comentario de los resultados obtenidos del análisis de las entrevistas, se hace necesario insistir, una vez más, sobre su carácter hipotético y provisional y el hecho de que su validez se limita exclusivamente a la 
muestra escogida. En ningún caso son extrapolables a otros grupos sociales (con otras edades, niveles de estudios, origen familiar, étnico, etc.). Su lectura debe impulsar el diseño de nuevas investigaciones que validen las hipótesis que aquí se plantean o proporcionar aportaciones conceptuales a nuevos modelos teóricos. Aclarado este punto, la conclusión principal se puede resumir de la siguiente forma: la influencia de los padres en la construcción de las trayectorias de género es más limitada de lo que se podría esperar a partir de lo propuesto por las explicaciones clásicas de la socialización. Pero esta afirmación necesita ser elaborada de forma pormenorizada. Empezando por el comentario de al gunas de las características de las dos generaciones que protagonizan nuestro estudio. $\mathrm{H}$ ay que tener en cuenta que entre ambas generaciones existe un gran sal to en los niveles de formación. Los padres han tenido una escasísima formación ( «yo sé leer y escribir», es la expresión que usan corrientemente para definir su nivel de estudios). Son excepcionales aquéllos que han llegado a la enseñanza secundaria. Por el contrario, una buena parte de sus hijos (puesto que se ha elegido a aquellos jóvenes que estaban matriculados en bachillerato) ha ido a la universidad. Además, entre las hijas y las madres de estas generaciones se observa un segundo salto. Las hijas cuentan con un trabajo asalariado y las madres han consagrado su vida al ejercicio de actividades domésticas y no asalariadas. También se podrían comentar otro tipo de diferencias entre las dos generaciones: una creció en el franquismo y la otra durante el inicio de la democracia, una se incorporó al mundo laboral en los años del boom económico y la otra durante el actual periodo de reestructuración económica, etc.

Estos saltos entre la generación de los padres y la de los hijos abren lo que se podría denominar una situación especial de transmisión. En efecto, cuando la investigación se encontraba en su primera fase, se pensaba que los padres podían tener una gran influencia en la configuración de las trayectorias escolares, familiares y profesionales de sus hijos e hijas. Sin embargo esto no ha sido así.

a) La trayectoria educativa. Por lo que se refiere a las trayectorias escolares, los padres han tenido un escaso papel. Los hijos no han seguido los estudios de sus padres. La nueva generación simplemente ha estudiado mucho más que la precedente. Pero los padres tampoco han podido tomar decisiones o aconsejarles sobre las opciones a seguir. D e hecho, cuando se les pregunta sobre las motivaciones de cualquier opción escolar (entre BUP y FP, entre ciencias y letras, el tipo de estudios universitarios, la decisión de acabar los estudios), ellos mencionan muy rara vez a sus padres. Y cuando se les pregunta de forma explícita sobre qué dijeron sus padres a propósito de las decisiones que habían tomado, la respuesta más general es del tipo: «mis padres me dejaban». Esta situación se explica por diferentes razones. La principal es el hecho de que los padres no pueden formarse una buena opinión por el simple hecho de que no conocen el sistema educativo (son mayores, no han estudiado). Como decía uno de los padres: 
«yo poco les he podido ayudar porque, se puede decir que en el primer curso ya me han superado en lo, ellos ya sabían más que yo. Así que ¿quién les iba a orientar? Si eran ellos los que me tenían que, en ese aspecto, orientarme a mí. Así que en la cultura simplemente estar pendientes de ellos. Eso sí».

b) La trayectoria profesional. El papel de los padres en la orientación profesional tampoco ha sido más importante. Dos hechos principales lo demuestran: ninguno de los hijos continúa la profesión de los padres y, en el caso de las mujeres, se pasa de madres amas de casa a hijas que sitúan su actividad profesional entre sus prioridades vitales. $Y$ un tercer hecho lo complementa. Los padres no han podido ayudar, más que de forma muy leve, a que ellos se incorporen a la vida laboral. La mayoría de los empleos han sido conseguidos sin la ayuda de los padres. Esto se explica por varias razones:

- Las redes sociales de los padres son bastante reducidas. La madres son amas de casa y muchos de los padres ya se han jubilado.

- Las redes sociales de los padres no son útiles para los hijos. Éstos se mueven en medios profesionales diferentes a los de los padres debido a su formación y a su orientación profesional. Esto es, medios sociales en los que los padres no conocen a nadie.

- Los padres, además, son de clase trabajadora y por consiguiente no conocen decisores (empresarios, cuadros directivos... ).

- Por último, tampoco han podido ayudarles a instalarse por cuenta propia. No tienen un negocio familiar que puedan cederles o no cuentan con suficientes recursos económicos.

En consecuencia, pese al importante peso que se suele atribuir al grupo familiar en las estrategias de inserción de los individuos en los puestos de trabajo, éste no es muy visible en el caso de aquellas familias en las que se ha producido un sal to generacional importante en los niveles educativos. Se ha de tener en cuenta que dicho salto afecta a una gran proporción de familias españolas en la actualidad, lo que puede estar explicando las grandes dificultades que encuentran muchos jóvenes para iniciar su vida profesional.

c) La trayectoria familiar. Por lo que se refiere a la trayectoria familiar, el modelo de vida familiar que han ofrecido los padres no podía resultar totalmente válido a sus hijos y, sobre todo, a sus hijas. Si entre la generación anterior el reparto de las obligaciones familiares quedaba claramente definido debido a que la mujer era ama de casa y no tenía responsabilidades fuera del hogar, entre la generación joven ninguna de las mujeres ha adoptado el papel de ama de casa. Los roles familiares entre una generación y la siguiente también han tenido que cambiar. Ello ha provoca- 
do interesantes novedades en al gunas de las parejas (con fórmulas bastante igual itarias), aunque las mujeres sigan siendo claves en las tareas de reproducción.

Pero si lo ocurrido en el interior de la familia no resulta satisfactorio para explicar por qué la situación social de hombres y mujeres continúa siendo diferente, ¿qué puede hacerlo? La investigación se centró en el estudio de las relaciones familiares, sin embargo, se preocupó igualmente por la influencia del sistema educativo y del mercado de trabajo.

\section{El sistema educativo}

Para los jóvenes entrevistados la influencia del sistema educativo parece haber sido determinante.

Los datos estadísticos disponibles sobre educación muestran con claridad que hombres y mujeres no se encuentran en condiciones de igualdad en el interior del sistema educativo. A pesar de que la prolongación de la escolarización ha sido más notable en el caso de las mujeres que en el de los hombres, ello no significa que se haya producido una democratización del sistema de enseñanza. La diferencia más notable es el hecho de que los hombres y mujeres cursan (en proporción) especialidades distintas, siendo las especialidades cursadas mayoritariamente por mujeres las que suelen ser menos prestigiosas. Este hecho plantea la cuestión sobre los mecanismos de selección internos al sistema educativo. Entre dichos mecanismos de selección se pueden incluir: los sistemas de calificación, los recursos económicos o la proximidad al centro de estudios. El análisis de las entrevistas sugiere algunas hipótesis interesantes sobre la forma que dichos mecanismos pueden estar beneficiando a unos 0 a otras. En cualquier caso, dado que una investigación sobre estos mecanismos necesitaría una técnica de carácter más «estructural» que los relatos de vida, se impone la prudencia metodológica y sugiere la necesidad de completar este punto con estudios especialmente diseñados para clarificar estos elementos.

Lo que sí puede ser detectado a partir de entrevistas son algunas de las representaciones sociales sobre el papel de la educación. U no de los elementos detectados es la contribución del sistema educativo en la imagen que los padres se hacen de sus hijos. Por ejemplo, una de las entrevistadas nos cuenta como su madre fue cambiando su forma de plantearse la educación de sus hijas. Al principio la madre tenía una idea muy tradicional: era el hijo el que debía estudiar más, porque al fin y al cabo es el hombre el que tiene que echar las cosas adelante. Pero a medida que la madre (a partir de los resultados escolares) va descubriendo que su hijo no muestra ninguna habilidad para los estudios y que, sin embargo, sus hijas son buenas estudiantes, va cambiando sus ideas. La madre acabó motivando a sus hijas a que estudiaran, y ellas ciertamente estudiaron bastante más que su hermano. Este ejemplo muestra como la propia imagen que ha creado el sistema educativo ha cambiado las expec- 
tativas y planes que la madre se hacía sobre sus hijos e hijas, Ilevándole incluso al abandono de sus ideas anteriores. En general, en todas las entrevistas a los padres se observó que el fracaso o el éxito en los estudios explicaban las expectativas que los padres se formaban sobre sus hijos e hijas con independencia de su sexo.

La formación influye además en la imagen que se tiene de los hombres y mujeres en el mercado de trabajo. En general se puede señalar que a medida que la formación aumenta, disminuye la importancia de la pertenencia de género. Esta disminución de la importancia de la pertenencia de género conforme aumenta el nivel de formación puede estar motivada por el hecho de que a medida que una persona adquiere una mayor educación empieza a ser más valorada por las capacidades que ha aprendido, mientras que las personas sin formación son valoradas haciendo uso de las categorías disponibles. Y evidentemente el hecho de ser hombre o mujer es uno de los criterios más claros que puede utilizar un empleador cuando carece de otro tipo de referencias.

Una escolarización prolongada también varía la propia percepción que las mujeres tienen de sí mismas. Actúa como un incentivo para su incorporación al mercado de trabajo, ya que gracias a su mayor cualificación pueden ocupar una mejor posición en el mismo y a que resulta muy satisfactorio poder aplicar los estudios una vez que se han terminado. Ellas en las entrevistas manifestaban siempre una gran implicación con su formación y el deseo de trabajar «en lo que he estudiado».

\section{El mercado de trabajo}

El mercado de trabajo constituye una segunda agencia de socialización determinante para comprender la diferencia entre hombres y mujeres. En nuestra muestra el trabajo de las mujeres está muy relacionado con el trabajo en el sector público (enseñanza, sanidad, servicios sociales, justicia) y, en menor escala, al trabajo en el sector privado. Se han sugerido varias explicaciones posibles: 1) los empleos en el sector público están más relacionados que los del privado con las carreras que han cursado las mujeres, 2) la actividades en el sector público se amoldan bien a los roles tradicionales de las mujeres, 3) este sector atrae a las mujeres porque no están discriminadas en el acceso, se les reconoce su formación, están mejor pagadas, sus derechos son respetados (en especial, la maternidad) y tienen mayor estabilidad. Por el contrario, el sector privado acaba expulsándolas mediante contratos precarios, la rele gación a cierto tipo de actividades, la condiciones de trabajo y los salarios.

Además, el mercado de trabajo se encuentra segmentado en función del género. H ombres y mujeres se reparten de forma desigual según el tipo de actividad. Según el tipo de labor a desarrollar se preferirá un hombre a una mujer. N ormalmente la diferencia en el tipo de actividad se traduce también en una diferencia en las condiciones de trabajo ( $M$ aruani, 1991).

Es especialmente destacable el hecho de que las mujeres entrevistadas sitúan la vida profesional como algo prioritario frente a otro tipo de alter- 
nativas vitales. Todas ellas han retrasado el momento de tener hijos. En concreto sólo dos de las entrevistadas habían tenido o iban a tener un niño (ambas estaban embarazadas en el momento de la entrevista) y lo hacen una vez que su vida profesional está asentada. Las demás todavía no han pensado en tenerlos, no quieren tenerlos o esperan a que se consolide su situación profesional. En este sentido, no se puede decir que hasta el momento (a la edad de 30-32 años en las mujeres entrevistadas) su trayectoria familiar haya limitado sus posibilidades profesionales. Tampoco se ha podido detectar en sus contestaciones que realizaran menores esfuerzos para encontrar empleo. Lo que sí se observa es que se emplean estrategias diferentes: las mujeres insisten más en la preparación de oposiciones y confían más en la formación como llave para el empleo. Todo ello consolida la idea de que la consecución de una mayor igualdad de género depende fundamentalmente de cambios en el funcionamiento del mercado de trabajo.

U na vez dicho esto, conviene discutir uno de los aspectos más controvertidos de esta investigación. M e refiero al hecho de haberla centrado en los años de transición a la vida adulta. Ello puede haber llevado al olvido de la importancia de la infancia en la formación de la identidad de los chicos y chicas. D e hecho se ha podido observar que al gunos de los comportamientos detectados probablemente tenían su origen en lo que había ocurrido en esos años anteriores. Por ejemplo, la interiorización de las responsabilidades domésticas, los factores que intervienen en la decisión sobre el tipo de estudios a seguir o la diferente forma de orientarse hacia el mundo del trabajo. Todos estos aspectos ejercen una poderosa influencia sobre el comportamiento de hombres y mujeres cuando forman su identidad profesional y familiar, o si se prefiere, ésta no se forma sólo durante los años de entrada a la vida adulta, sino que se ha ido configurando a lo largo de toda la vida. N o obstante, lo que este trabajo sugiere es que, si bien el peso todas estas determinaciones de origen familiar es muy fuerte, no son determinantes. U nas condiciones concretas del mercado de trabajo o una mejora en los niveles de formación pueden hacer, por sí solos, y esto es lo importante, que los efectos de esa determinación desaparezcan 0 , al menos, sean minimizados. U na niña que nazca en el seno de una familia en la que la madre sea el arquetipo de ama de casa no acabará necesariamente como tal. Bastará con que tenga una mayor escolarización que la que tuvo su madre y que encuentre cierta facilidad para entrar en el mercado laboral para que el modelo materno deje de hacer sentir su influencia. Evidentemente, no habrá borrado todo lazo con su progenitora. Seguramente siga compartiendo muchos rasgos (en su forma de hablar, de organizar las tareas del hogar), pero tendrá una posición social radicalmente diferente a la que ocupa su madre. Será una mujer activa que entiende la vida familiar y profesional de forma total mente distinta a como lo hacía aquélla.

En definitiva, el cambio de la situación social de la mujer puede producirse incluso en el caso de que la socialización de roles masculinos y femeninos en el interior de las familias no cambiara. Sería suficiente con el hecho 
de que otras instituciones alteraran su forma de entender la relación con las mujeres. Algunos de esos cambios se han producido de forma más o menos avanzada. Los mejores ejemplos de ello son la mejora de las posibilidades educativas de las mujeres y la mejora de las condiciones del mercado de trabajo femenino, sobre todo gracias a la extensión del sector público y la protección de ciertos derechos laborales. El estímulo o la restricción de este tipo de políticas tendrá sus efectos sobre evolución futura de la situación de las mujeres.

El diseño de una política social y de unos servicios sociales que liberen a la familia (y por tanto a la mujer) de parte de las labores reproductivas (cuidado de niños, de ancianos, de enfermos) puede ser uno de los grandes instrumentos para la consecución de nuevas formas familiares más igualitarias.

En algunos sentidos dichas formas familiares están empezando a construirse desde abajo. La forma en que las jóvenes actuales planifican su vida familiar y profesional está cambiando ya la forma de entender la familia. En las entrevistas las contestaciones de las mujeres reflejan una reflexión continua sobre la forma de plantearse el empleo y la vida familiar (lo que contrasta con el menor grado de reflexividad e innovación en las contestaciones de los varones). Reflexiones que ponen en práctica en la relación con sus compañeros. Las mujeres están teniendo un papel activo en la construcción de una nueva organización social distinta al patriarcado, pero todos esos esfuerzos pueden verse frenados, limitados e, incluso, invertidos si no se corresponden con cambios en el resto de instituciones sociales. Cambios que todavía deben afectar a la organización del mercado laboral y a la orientación de las políticas públicas.

\section{Bibliografía}

Battagliola, F. (1984). «Employés et employées. Trajectoires professionnelles et familial es». Le Sexe du Travail. Structures familiales et système reproductif, p. 57-70. Grenoble: PU G

Battagliola, F.; Brown , E.; J ASPARD, M . (1995). «Precarité d'emploi et itinéraires de transition à l'âge adulte». Recherches et Prévisions, monographique: Jeunesse: le plus bel âge de la vie?, núm. 40, junio de 1995, p. 45-56.

Baudelot, Ch.; Establet, R. (1992). Allez les filles! París: Éditions de Seuil.

BENERIA, L. (1991). «La globalización de la economía y el trabajo de las mujeres».

Revista de economía y sociología del trabajo, núm. 13-14, 1991, p. 23-35.

BERger, P.; LuCKMANN, Th. (1968). La construcción social de la realidad. Buenos Aires: Amorrortu, 1993.

BLöss, Th. (1997). Le liens de famille. Sociologie des rapports entre générations. París: Presses U niversitaires de France.

Casal Bataller, J.; M asjuan Codin A, J.M .; Planas Coll, J. (1991). La inserción social y profesional de los jóvenes. M adrid: CIDE.

Dem AZIĖRE, D .; D uBAR, C. (1997). Analyser les entretiens biographiques. L'exemple de récits d'insertion. París: N athan, colección Essais et Recherches. 
D ubar, C. (1991). La socialisation. Construction des identités sociales et professionne Iles. París: Armand Collin, colección U, serie Sociología.

D uRKH EIM , É. (1922). Educación y sociología. Barcelona: Ediciones Península, 1975.

ERIKSON , E.H . (1968). Adolescence et crise. La quête de l'identité. París: Flammarion, 1972.

García M ontalvo, J.; Palafox, J.; Peiró, J.M .; Prieto, F. (1997). Capital humano: la inserción laboral de los jóvenes en la Comunidad Valenciana. Valencia: IVIE, Fundación Bancaixa.

I gLESIAS DE U SSEL, J. (1998). La familia y el cambio político en España. M adrid: Tecnos, colección Semilla y Surco.

Kauffm AN N, J.C. (1994). «Rôles et identité: l'exemple de l'entrée en couple». Cahiers internationaux de sociologie, vol. XCVII, 1994, p. 301-328.

M ARUANI, M . (1991). «La construcción social de las diferencias de sexo en el mercado de trabajo». Revista de economía y sociología del trabajo, sept-dic. 1991, núm. 13-14.

Palafox, J.; Pérez, F.; Cubel, A.; Valero, S.; Villarreal, E. (1997). Capital humano. Educación y empleo en la Comunidad Valenciana. Valencia: Fundación Bancaixa-IVIE.

PlanAs, J.; CASAL, J.; BRullet, C.; M ASJuÁn, J.M . (1995). La inserción social y profesional de las mujeres y los hombres de 31 años. Barcelona: ICE, U niversitat Autònoma de Barcelona.

PN U D, Programa de las N aciones U nidas para el D esarrollo (1995). Informe sobre desarrollo humano, 1996. 